\title{
https://doi.org/10.46344/JBINO.2021.v010i01.17
}

\section{VASCULAR RESPONSES TO MANUAL ST36 ACUPUNCTURE IN HEALTHY SUBJECTS ASSESSED THROUGH A KINETIC STUDY}

\section{José Federico Rivas-Vilchis ${ }^{1 *}$, Fernando Hernández-Sanchez ${ }^{2}$, Moisés Salvador Castañeda-Ramírez'}

'Especialización en Acupuntura y Fitoterapia, División de Ciencias Biológicas y de la Salud, Unidad Iztapalapa, Universidad Autónoma Metropolitana, México.

¿Unidad de Materiales, Centro de Investigación Científica de Yucatán, AC. Mérida, Yucatán, México

E-mail: ifrv@xanum.vam.mx

\begin{abstract}
Objective: This study aimed to analyze the effect of acupuncture in Stomach 36 (ST36 or Zusanli) acupoint on cardiovascular function in healthy subjects. Design. A kinetics crosssectional experimental study was conducted at Clínica de Acupuntura, Unidad Iztapalapa, Universidad Autónoma Metropolitana, Ciudad de México, México. Subjects and methods: Ten healthy university students (four women) aged $24.3 \pm 2.16$ (mean \pm SD) participated in the present study to investigate the kinetics of the acute effect of acupuncture stimulation at ST36. The study consisted of one session of manual acupuncture in the ST36 acupoint in the right arm. The following data for continuous beatto-beat registration: mean arterial pressure (MAP), cardiac output (CO), heart rate (HR), stroke volume (SV), and total peripheral resistance (TPR) were computed. Results: This treatment showed a remarkable decreasing effect on CO, HR, and SV during the 60 seconds of acupuncture. Otherwise, TPR was increased. Conclusions: Data that manual acupuncture of Zusanli evoked a complex cardiovascular response probably related to a vagal response.
\end{abstract}

Keywords: ST36 acupoint; mean arterial pressure, cardiac output; heart rate; stroke volume; total peripheral resistance. 


\section{INTRODUCTION}

Experimental laboratory studies have demonstrated that acupuncture modulates neurohumoral regulatory systems and modify cardiovascular function (Uchida et al., 2108; Armstrong et al., 2020; Cheng 2014). Many researchers have used the acupunctural stimulus as a non-invasive stimulus and assess the autonomic nervous system and study the heart rate variability among other cardiovascular variables (Lee et al., 2010, Rivas-Vilchis et al., 2019). According to its name in Chinese medicine, acupoint Stomach 36 (ST36) or Zusanli is frequently used to treat cardiovascular disorders (de Lima Pimentel et al., 2019). It has been reported that acupuncture in ST36 improves prehypertension and hypertension (Li et al., 2015); and modify the circadian rhythms of blood pressure (Kim et al., 2012). Moreover, stimulation of ST36 in combination with other acupoints showed beneficial effects in patients with angina pectoris (Richter et al., 1991). The
ST36 acupoint lies laterally and inferiorly to the anterior tibial spine, and it is related to the anterior tibialis muscle and deep peroneal nerve (Cheng and Wang YP, 2002); see Figure 1. Our study aimed to analyze the kinetics of the immediate cardiovascular response to manual acupuncture in ST36 in healthy subjects.

The Finapres finger cuff recording system allows to immediately, continuously, and non-invasively visualize changes in mean arterial pressure (MAP), heart rate (HR), stroke volume (SV), cardiac output (CO), and total peripheral resistance (TPR) through continuous calculations of beatto-beat variations in experimental subjects (Finapres, 2002). This system allows us to examine changes in these variables at rest or during movement (Hodgson and Choate 2012; Hill et al., 2013; MacEwan et al., 2018); therefore, this system could continuously assess the effect of acupuncture interventions.

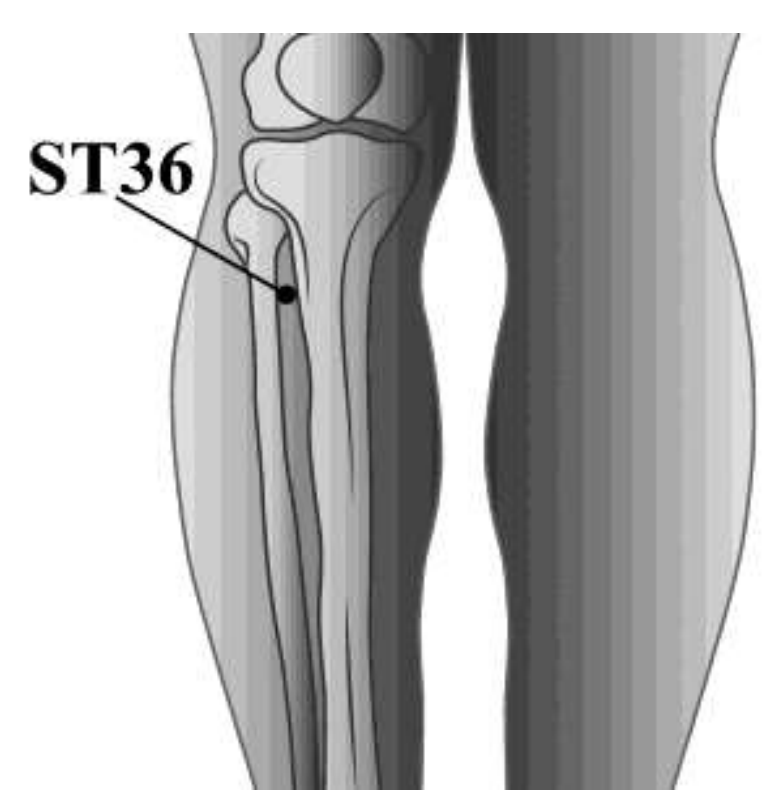

Figure 1. Stomach 36 acupoint (St36 or Zusanli) 


\section{MATERIALS AND METHODS}

Study Design and setting. A transversal and kinetic study without control was conducted. The study was performed at Acupuncture Clinic, Unidad Iztapalapa, Universidad Autónoma Metropolitana.

Population. All of the subjects in this study were healthy student volunteers of the Institution Universidad Autónoma Metropolitana, campus Iztapalapa, Ciudad de México, México. These subjects were recruited by advertising. The study population consisted of 1014 female) healthy subjects having a global age of $24.3 \pm 2.16$ (mean $\pm S D$ ), without clinical data of cardiovascular diseases, obesity, athletic training; non-smoking, and without having received medication or treatment with acupuncture in the ten days before the study.

Ethical aspects. The institutional Biological and Health Division Ethics Committee approved this study (Approval Number 15.04.16). The study conforms to the principles of the revised version of the Declaration of Helsinki (World Medical Association 2013). The subjects gave written, informed consent to participate.

\section{Instrumentation and recordings}

A continuous beat-to-beat blood pressure (BP) data from our sample of 10 subjects was used in the present study. A finger cuff was placed in the middle phalanx of the left middle finger, with the hand positioned at heart level (Finometer; Finapres Medical Systems, Amsterdam, The Netherlands), and arterial pressure was recorded continuously. The Finapres system provides continuous and concurrent calculations of MAP, CO, TPR, HR, and SV and is commonly used in research settings (Chin and Panerai, 2012). Participants completed a supine resting recording period of $5 \mathrm{~min}$. Data recorded estimate hemodynamic parameters using the Modelflow method based on a threeelement Windkessel model (Finapres, Beatscope). The MAP, CO, HR, and SV were calculated by BeatScope - v02.10 software (Finapres Medical Systems, Enschede, The Netherlands). This software allows online monitoring, control, storage, and offline revise of the complete Finometer data, including cardiac parameters (Hill et al., 2012). The TPR was determined as the quotient of ModelFlow-derived MAP divided by CO. The unit of measurement for ModelFlow TPR is CGS units, i.e., dys.s. $\mathrm{cm}^{-5}$.

\section{Signal processing and analysis}

Initially, beat-to-beat blood pressure (BP) signals were smoothed (FFT Smooth) to reduce low-frequency baseline variation. This type of smoothing is designed to reduce the artefactual time delays or distortion to the waveforms and facilitate reliable recognition of pulse landmarks at all sites (Ifeachor and Jervis, 1993). Ectopic beats were also excluded, and any low-quality pulse with visible movement artifact, noise, or incorrect landmark recognition was discarded. After that, all recorded signals from each experimental run were plotted and then averaged with the software Plot2 for Mac (Michael Wesemann, Berlin, Germany, 2019).

\section{Acupuncture treatment}

Manual acupuncture without additional electrical or laser stimulation was applied 
to the ST 36 acupoint. ST36 is located on the lateral side of the stifle joint adjacent to the anterior tubercle of the tibia, Figure

1. The right leg point leg was traditionally located wherein the needle was inserted vertically to a depth of $2.0 \mathrm{~cm}$ for 60 seconds. Sterile acupuncture $40 \mathrm{~mm}$ long, $0.25 \mathrm{~mm}$ long, with a silver handle (Seirin Corporation, Shizuoka, Japan) were used.

Practitioner's Background. One acupuncturist, with six years of experience, performed all the acupuncture stimuli.

\section{Study Protocol}

The subjects were instructed to abstain from alcohol for $\geq 24 \mathrm{~h}$ before the experiment; coffee, tea, and exercise on the day of experiments, and to have a light meal two $h$ before each experiment.
During the study, the subjects remained supine. After registration of its clinical information, each subject was at rest for $\geq 15$ min before starting the records. The measurements were taken between 9:00 a.m. to 12:00 p.m. to standardize the circadian variations in blood pressure. After the rest period, the Finapres finger cuff was affixed to the middle finger of each subject. Data recording commenced, and after five-minutes of registration, acupuncture in the right ST36 acupoint was applied for an additional record of 60 seconds. After the needle was removed, the recording was continued during additional five-min period, Figure 2. This study was ten records with the same experimental protocol, performed in the same laboratory. The outcome variables were: MAP, CO, TPR, HR, and SV.

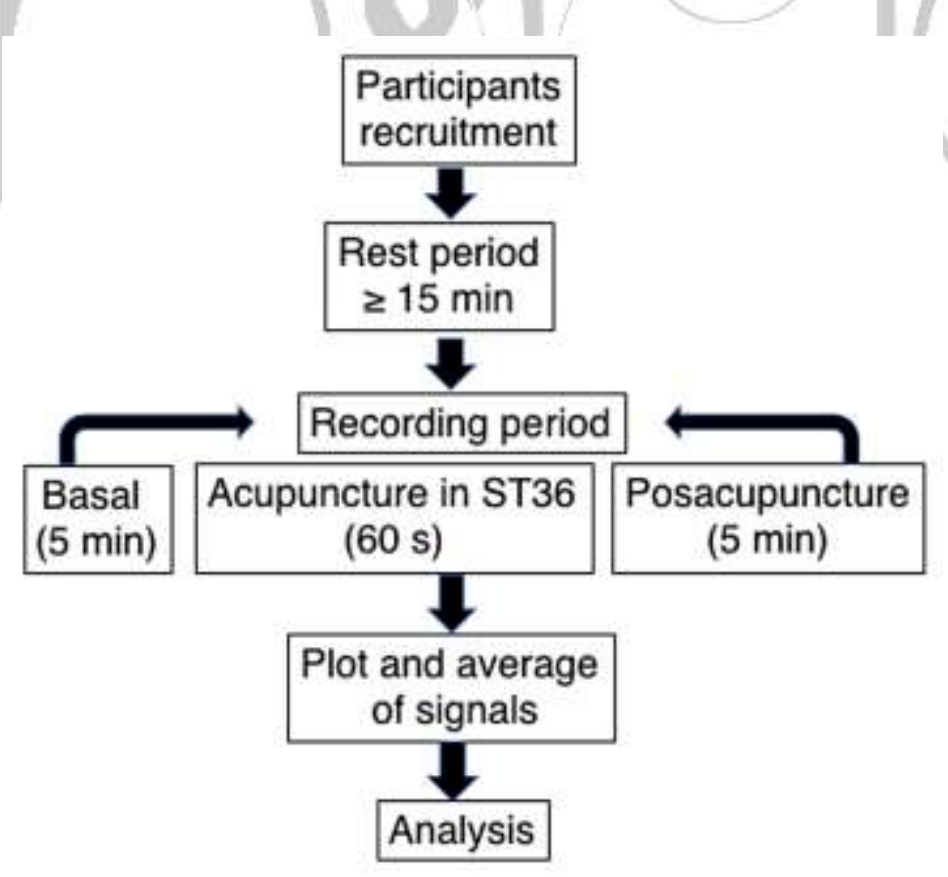

Figure: Flow chart of Study 


\section{Comparisons and statistics}

The records of each subject in the total period of 11 minutes were plotted in the Plot2 software (Michael Wesemann, Berlin, Germany, 2019). Interpolated data for the ten subjects were averaged, and a kinetic curve of the acupuncture effect was obtained for every parameter. Subsequently, the curves corresponding to each variable were gathered in a single graph. The data before acupuncture $(\min 3)$ and at the end of registration ( $\min 11)$ for each variable were compared with the t-student of the two-tail test. The data were statistically analyzed, using the statistical package for the social sciences (SPSS, version 22.0) (Chicago, USA). Data are indicated as means $\pm S D$. The level of statistical significance was $p<0.05$.

\section{RESULTS AND DISCUSSION}

Table 1 shows cardiovascular changes elicited through manual acupuncture in ST36. Comparing the basal ( $\min 3$ ) versus the end of the post acupuncture experimental period ( $\min 11)$ : TPR increased significantly, $p=0.036$. Otherwise, CO and SV decreased significantly, $p=0.027$ and $p=0.041$, respectively. Although MAP and HR initially diminished after the acupuncture treatment, at the end of the 11-minute experimental period showed a nonsignificant decrease, $p=0.306$ and $p=$ 243 , respectively.

\begin{tabular}{|c|c|c|c|}
\hline Parameters & $\begin{array}{c}\text { Basal } \\
(\min 3)\end{array}$ & $\begin{array}{l}\text { Pos-treatment } \\
(\min 11)\end{array}$ & p-value \\
\hline MAP (mmHg) & $79.2+12.3$ & $764+114$ & 0.306 \\
\hline $\mathrm{CO}(\mathrm{L} / \mathrm{min})$ & $5.9 \pm 1.2$ & $4.78 \pm 0.89$ & $0.027 *$ \\
\hline $\begin{array}{l}\text { TPR } \\
(\text { dyn.s/cm-5) }\end{array}$ & $907.8 \pm 190.2$ & $1105.6 \pm 232.2$ & $0.036^{*}$ \\
\hline HR (beat/min) & $72.9 \pm 6.6$ & $69.4 \pm 6.1$ & 0.243 \\
\hline $\mathrm{SV}$ (ml/beat) & $77.4 \pm 17.8$ & $62.3 \pm 13.1$ & $0.041 *$ \\
\hline \multicolumn{4}{|c|}{$\begin{array}{l}\text { Mean } \pm \text { standard deviation. } \\
\mathrm{MAP}=\text { Mean arterial pressure; } \mathrm{CO}=\text { Cardiac output } \mathrm{TPR}=\text { Total peripheral } \\
\text { resistance; } \mathrm{HR}=\text { Heart rate; } \mathrm{SV}=\text { Stroke volume. } \\
{ }^{*} \mathrm{p}<0.05\end{array}$} \\
\hline
\end{tabular}

Table 1. Comparison of cardiovascular parameters before and after manual acupuncture in Stomach 36 acupoint.

Kinetics study

Effect of manual acupuncture at ST36 on mean arterial pressure and its variables
The continuous and non-invasive cardiovascular parameters measurement is suitable for a better understanding the 
immediate effects and mechanisms of acupuncture (Rivas-Vilchis et al., 2020). cardiac cycle. MAP is related to changes in CO and TPR; in turn, the CO and the TPR have their determinants. The beat-tobeat temporal variations of MAP and its $\mathrm{CO}$ and TPR variables after acupuncture Mean arterial pressure (MAP) is the are shown in Figures 3 and 4. average blood pressure throughout a

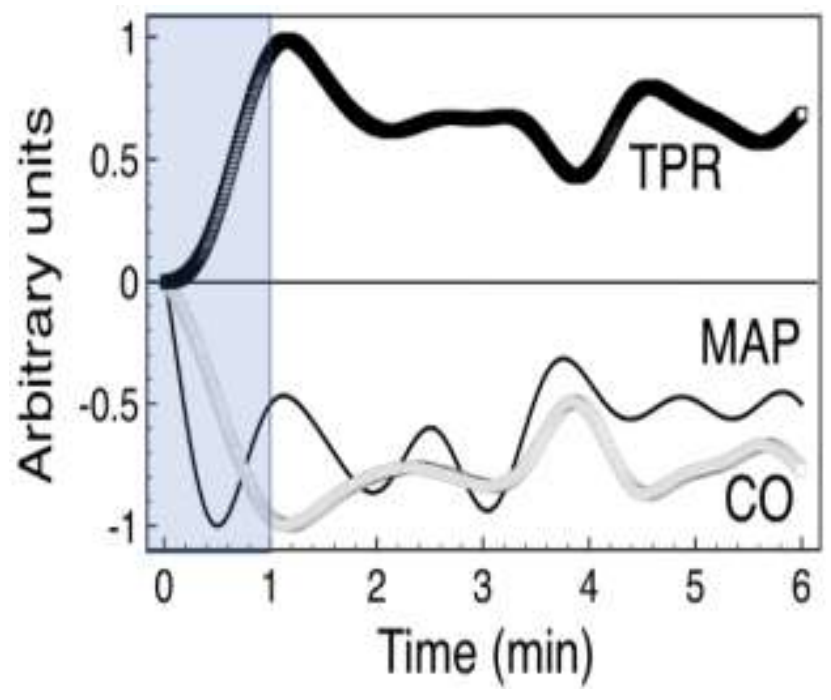

Figure 3 - The data shown is an average of the 6-minutos recordings of a continuous beat-to-beat period of mean arterial pressure (MAP), cardiac output (CO), and total peripheral resistance (TPR) of all participants. Shaded area depicts one-minute of manual acupuncture in ST36 acupoint.

Figure 3 shows the changes in MAP, CO, and TPR during the 6-min following the manual acupuncture of ST36, and Figure 4 shows the 60 seconds of acupuncture, respectively. Acupuncture in ST36 produced only a marginal decrease in MAP despite a decrease in CO because of a noticeable compensatory TPR increase, Figure 3. 


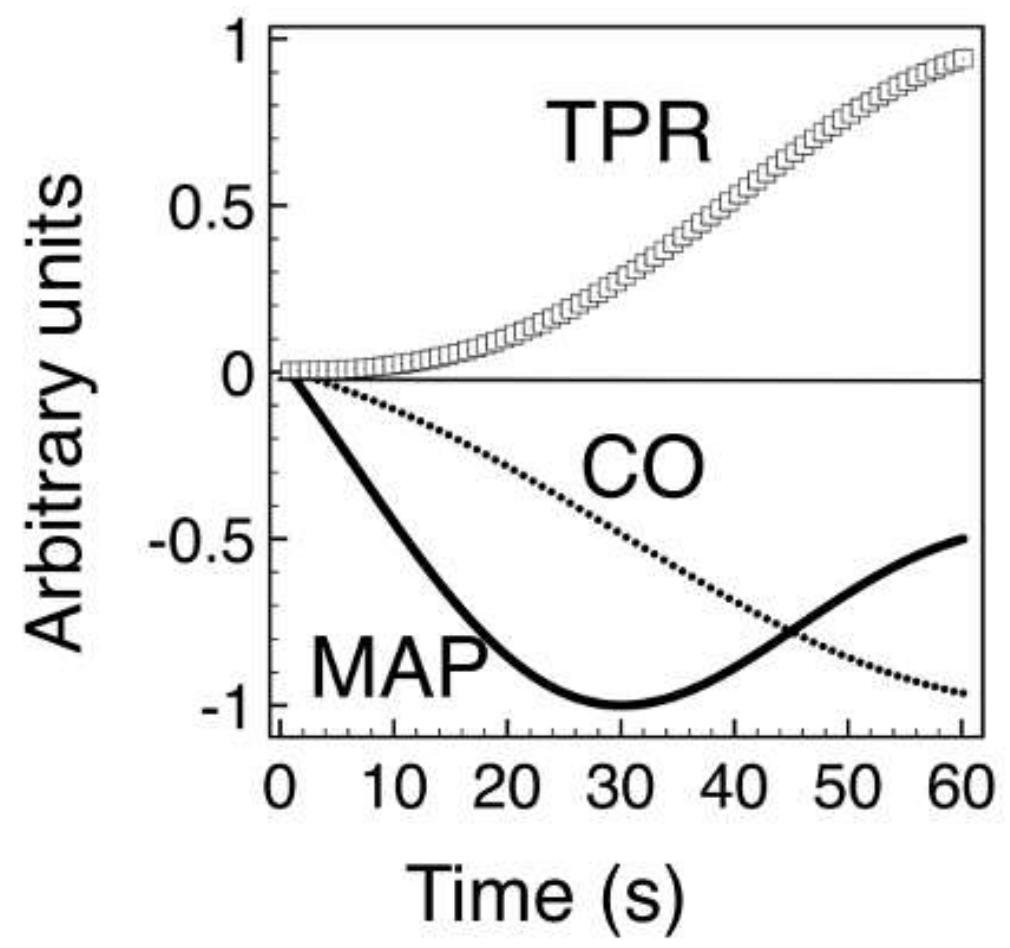

Figure 4. The data shown is an average of the 60-seconds recordings of a continuous beat-to beat period of mean arterial pressure (MAP), cardiac output (CO), and total peripheral resistance (TPR) of all participants during acupuncture in ST36.

The changes in MAP, CO, and TPR during the 60-second acupuncture period are shown in Figure 4. It is observed that $\mathrm{CO}$ presented a continuous decrease during this period. At the same time, the TPR presented an S-type growth during this same period. The increase in TPR corresponds initially with a decrease in $\mathrm{CO}$, during the second part, with an increase in MAP. Otherwise, MAP presented a biphasic behavior with an initial decrease related to the $\mathrm{CO}$ decrease in $\mathrm{CO}$ and secondary compensation related to a TPR increase.

There has been a description of the inhibitory effects of electroacupuncture of the Zusanli acupoint on the cardiovascular system, including reductions in arterial pressure, HR (Chiu and Cheng, 1974), and sympathetic nerve activity (SNA) (Yao, 1993).

Effect of manual acupuncture at ST36 on cardiac output and its variables

$\mathrm{CO}$ is the volume of blood the heart pumps per minute and is calculated as the heart rate and stroke volume product. The beat-to-beat temporal variations of $\mathrm{CO}$ and its $\mathrm{HR}$ and $\mathrm{SV}$ variables following acupuncture stimulation are shown in Figures 5 and 6 . Figure 5 shows the changes in $\mathrm{CO}, \mathrm{HR}$, and SV following the manual acupuncture of ST36, and Figure 6 shows the 60 seconds of acupuncture, respectively. 


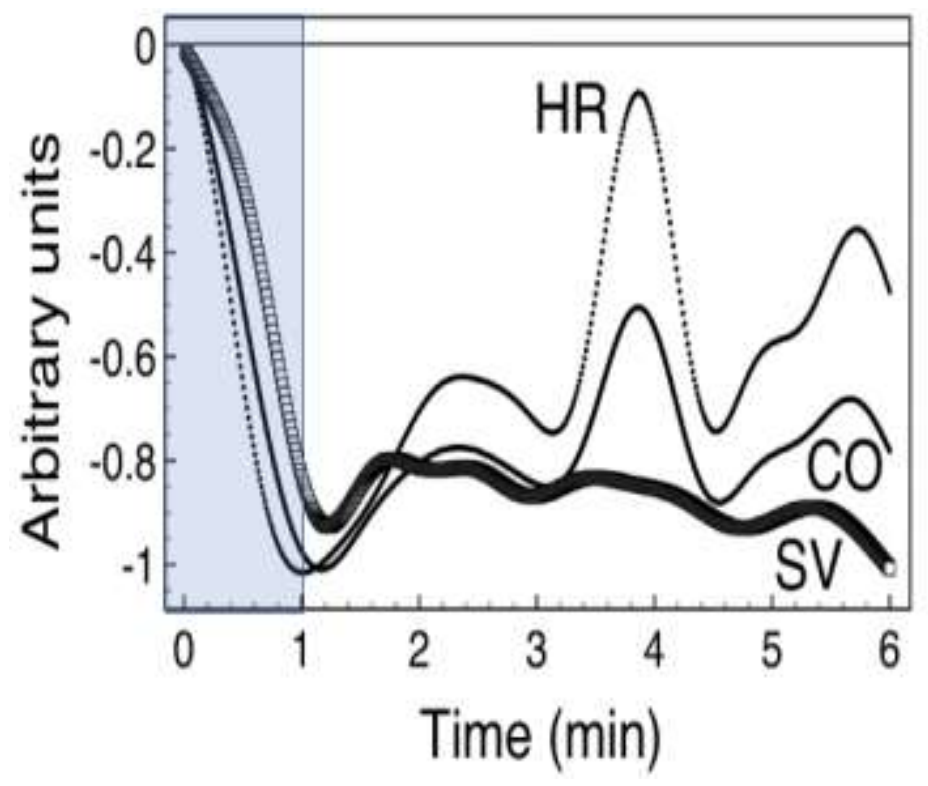

Figure 5. The data shown is an average of the 6-minutes recordings of a continuous beat-to-beat period of cardiac output $(\mathrm{CO})$ and its variables: heart rate (HR) and stroke volume (SV) of all participants. The shaded area depicts one-minute of manual acupuncture on ST36 acupoint.

$\mathrm{CO}, \mathrm{HR}$, and SV decreased markedly during the 60 seconds of acupuncture stimulation. SV presented the most persistent decrease, Figure 5.

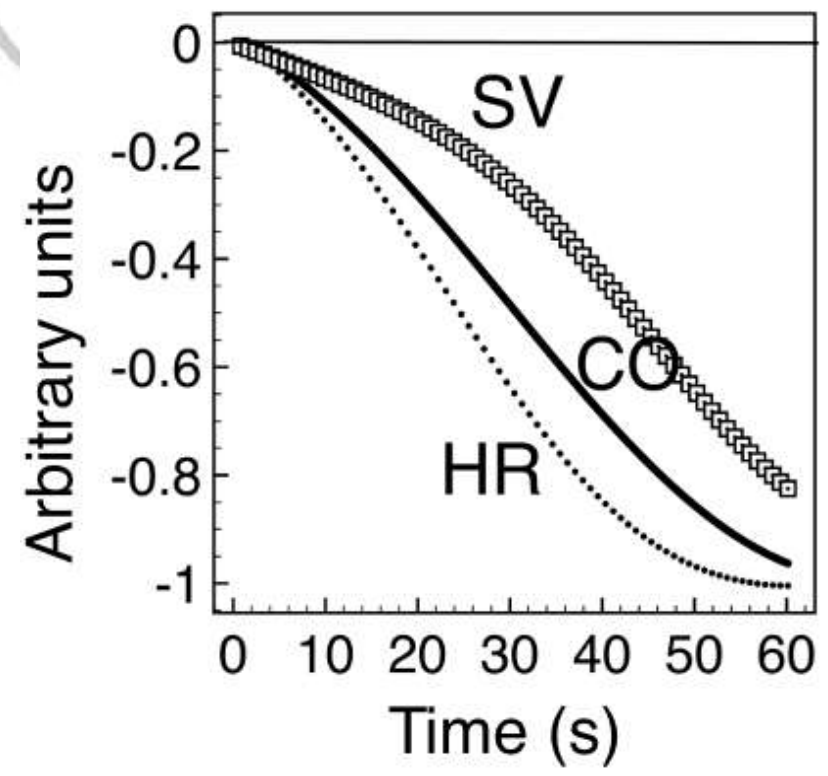

Figure 6. The figure shown are an average of the 60-seconds of a continuous register signal of the cardiac output $(\mathrm{CO})$ and its variables: heart rate $(\mathrm{HR})$, and stroke volume of all participants during acupuncture in ST36. 
The changes in $\mathrm{CO}, \mathrm{HR}$, and SV during the 60 seconds period during acupuncture are shown in Figure 6. HR and SV steadily decreased, and therefore CO diminished. Otherwise, SV presented an S-type decrease during this same period. SV is determined by ventricular inotropy and preload or end-diastolic volume. Preload is affected by blood volume and the compliance of veins. Decreasing the blood volume decreases the preload, decreasing the SV, and therefore decreasing CO. Vagal stimulation has been shown to decrease $H R$ and alters the loading conditions of the left ventricle (LV) (Xenopoulos and Applegate, 1994). Our results are consistent with previous studies suggesting that stimulation of ST36 evoked a vagal response (Ouyang et al., 2002; Ye et al., 2006), and sympathetic inhibition (Ernst and Lee, 1986; Michikami et al., 2006)

\section{Time sequence of cardiovascular changes}

The first derivative of each signal (dy/dt) was used to identify the time and sequence of the maximum speeds of the initial changes in each variable during acupuncture. Therefore, the first derivate of each 20-seconds signal after needling was calculated. Figure 7 depicts the time sequence of $\mathrm{dMAP} / \mathrm{dt}$, $\mathrm{dCO} / \mathrm{dt}$, and dTPR/dt elicited by acupuncture in ST36. The MAP derivative had a biphasic behavior with a small increase and a sharper decrease with speed changes in the seconds 6.1 and 13.0 seconds, respectively, Figure 7 . On the other hand, CO showed a decrease with a maximum speed at 12.2 seconds. Otherwise, TPR showed an increase with a maximum speed at 12.2 seconds.

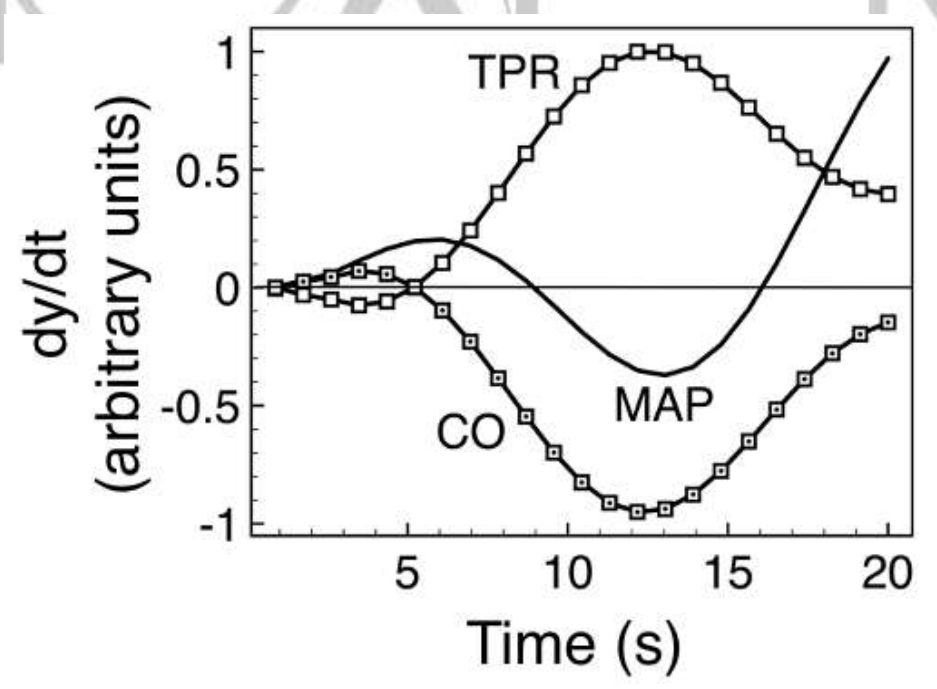

Figure 7. First derivative trace of mean arterial pressure (MAP), cardiac output (CO), and total peripheral resistance (TPR) during the first 20-seconds of acupuncture stimulation of ST36. All traces represent the average of the signal of all participants. 
Figure 8 depicts the time sequence of $\mathrm{dCO} / \mathrm{dt}$, dHR/dt, and dSV/dt. Both CO and HR showed a decrease with maximum speed at 12.2 seconds. Otherwise, the SV first derivative showed a biphasic shape with an increment followed by a decrease with maximum speed at either 6.1 and 14.8 seconds, respectively.
The first derivative of SV showed a biphasic behavior with a successive increase - decrease with maximum speeds at 6.1 and 14.8 seconds. Otherwise, $\mathrm{CO}$ and HR decrease with maximum speed at 12.2 seconds, Figure 8.

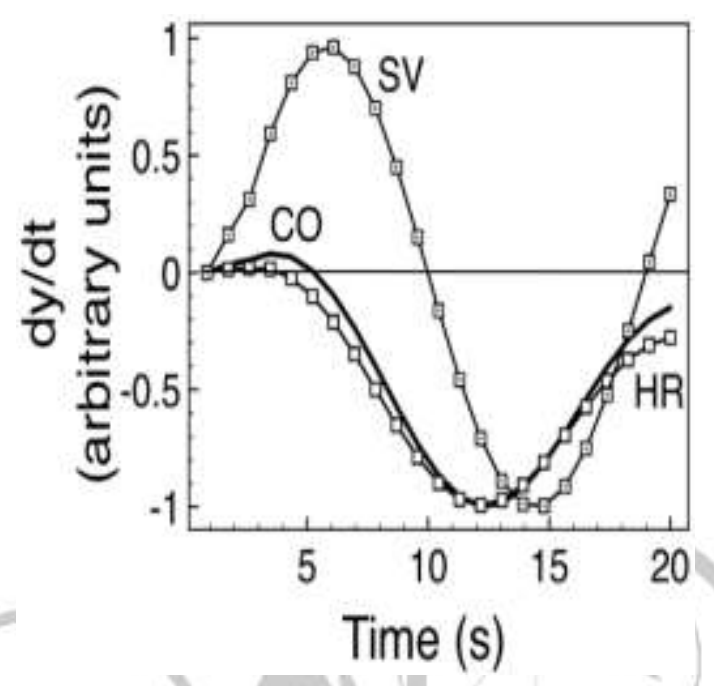

Figure 8. First derivative trace of cardiac output $(\mathrm{CO})$, heart rate (HR), and stroke volume (HV) during the first 20-seconds of acupuncture stimulation on ST36. All traces represent the average of the signal of all participants.

\section{Summary of results}

- Comparing basal and post acupuncture periods, acupuncture in ST36 caused in both CO, SV significant decrease, and a TPR significant increase.

- The kinetic study of MAP and its variables $\mathrm{CO}$ and TPR showed that MAP and CO decreased, and TPR increased markedly during the period of stimulation with ST36.

- TPR showed a type $S$ shape during the period of stimulation with acupuncture.

- The kinetic study of $\mathrm{CO}$ and its variables $H R$ and SV showed that HR and SV decrease, and consequently, CO decrease during the period of stimulation with ST36. 
- SV showed a type S shape during the period of stimulation with acupuncture.

- The analysis of the speed in the circulatory changes elicited by acupuncture during the initial 20 seconds showed that: MAP and SV slight increase and later decrease, and both $\mathrm{CO}, \mathrm{HR}$ decreased. Besides, MAP showed a decrease with a maximal speed concordance with the $\mathrm{CO}$ decrement and TRP increment.

\section{CONCLUSION}

Manual acupuncture of ST36 HR steadily decreased HR and sharply increased TPR. Otherwise, decreased $\mathrm{CO}$ accordingly to $H R$, whereas increased the TPR in a manner probably related to a compensatory response. In conclusion, manual acupuncture of Zusanli evoked a complex cardiovascular response, probably related with a vagal response.

\section{CONFLICT OF INTEREST}

The authors have no conflict of interest to declare.

\section{BIBLIOGRAPHY}

Armstrong K, Gokal R, Todorsky W. Neuromodulating Influence of Two Electroacupuncture Treatments on Heart Rate Variability, Stress, and Vagal Activity. J Altern Complement Med. $2020 \mathrm{Jul} 10$. doi: $10.1089 / \mathrm{acm} .2019 .0267$.
Cheng KJ. Neurobiological mechanisms of acupuncture for some common illnesses: a

clinician's perspective. J Acupunct Meridian Stud. 2014;7(3):105-14.

Cheng K, Wang YP. Color Atlas of Acupuncture Acupoints. Beijing: China Press of Traditional Chinese Medicine, 2002:312-18.

Chin KY, Panerai RB. Comparative study of Finapres devices. Blood Press Monit. 2012;17(4):171-8.

Chiu DTJ and Cheng KK. A study of the mechanism of the hypotensive effect of acupuncture in the rat. Am J Chin Med. 1974;2:413-19.

de Lima Pimentel R, Duque AP, Moreira $B R$, Rodrigues LF Junior. Acupuncture for the Treatment of Cardiovascular Diseases: A Systematic Review. J Acupunct Meridian Stud. 2019;12(2):4351.

Ernst $M$, Lee $M H$. Sympathetic effects of manual and electrical acupuncture of the Tsusanli knee point: comparison with the Hoku hand point sympathetic effects. Exp Neurol. 1986:94(1):1-10.

Finapres Medical Systems. BeatScope 1.1. User's Guide. Finapres Medical Systems; Amsterdam, the Netherlands: 2002.

Hill LK, Sollers III JJ, Thayer JF. Resistance reconstructed estimation of total peripheral resistance from computationally derived cardiac output - 
biomed 2013. Biomed Sci Instrum. 2013;49:216-23.

Hill LK, Sollers JJ lii, Thayer JF. Evaluation of a simple estimation method for the derivation of cardiac output from arterial blood pressure and heart rate. Biomed Sci Instrum. 2012; 48:165-70.

Hodgson Y, Choate J. Continuous and noninvasive recording of cardiovascular parameters with the Finapres finger cuff enhances undergraduate student understanding of physiology. Adv Physiol Educ. 2012;36(1):20-6.

Ifeachor EC, Jervis BW. Digital Signal Processing: a Practical Approach. 1993; Wokingham: Addison-Wesley.

Kim HM, Cho SY, Park SU, Sohn IS, Jung WS, Moon SK, Park JM, Ko CN, Cho KH. Can acupuncture affect the circadian rhythm of blood pressure? A randomized, double-blind, controlled trial. J Altern Complement Med. 2012;18(10):918-23.

Lee S, Lee MS, Choi JY, Lee SW, Jeong SY, Ernst E. Acupuncture and heart rate variability: a systematic review. Auton Neurosci. 2010;155(1-2):5-13.

Li P, Tjen-A-Looi SC, Cheng L, Liu D, Painovich J, Vinjamury S, Longhurst JC. Long-lasting reduction of blood pressure by electroacupuncture in patients with hypertension: Randomized controlled trial. Med Acupunct. 2015;27(4):253-266.

MacEwen C, Sutherland S, Daly J, Pugh C, Tarassenko L. Validation of modelflow estimates of cardiac output in hemodialysis patients. Ther Apher Dial. 2018;22(4):337-344.

Michikami D, Kamiya A, Kawada T, Inagaki $M$, Shishido T, Yamamoto K, Ariumi H, Iwase S, Sugenoya J, Sunagawa K, Sugimachi $M$. Short-term electroacupuncture at Zusanli resets the arterial baroreflex neural arc toward lower sympathetic nerve activity. Am J Physiol Heart Circ Physiol. 2006;291 (1):H318-26.

Ouyang $\mathrm{H}$, Yin J, Wang $Z$, Pasricha PJ, Chen JD. Electroacupuncture accelerates gastric emptying in association with changes in vagal activity. Am J Physiol Gastrointest Liver Physiol. 2002;282(2):G390-6.

Richter A, Herlitz J, Hjalmarson A. Effect of acupuncture in patients with angina pectoris. Eur Heart J. 1991;12(2):175-8.

Rivas-Vilchis JF, Hernández Sánchez F, Gámez-Valdés E. Determination of vascular responses to manual PC6 acupuncture in subjects healthy and diabetics by the second derivative of the finger photoplethysmogram waveform. Asian J Pharm Pharmacol 2019:5(5):97983.

Rivas-Vilchis JF, Hernández-Sánchez F, Castañeda-Ramírez MS. Kinetics of cardiovascular function changes elicited by auricular acupuncture in Heart point. Rev Int Acup (2020) 14(2):41-45.

Uchida C, Waki H, Minakawa Y, Tamai H, Hisajima T, Imai $K$. Evaluation of 
Autonomic Nervous System Function Using Heart Rate Variability Analysis During Transient Heart Rate Reduction Caused by Acupuncture. Med Acupunct. 2018;30(2):89-95.

Xenopoulos NP, Applegate RJ. The effect of vagal stimulation on left ventricular systolic and diastolic performance. Am J Physiol. 1994;266(6 Pt 2):H2167-73.

Yao T. Acupuncture and somatic nerve stimulation: mechanism underlying effects on cardiovascular and renal activities. Scand J Rehabil Med Suppl. 1993;29:7-18.

Ye $X$, Li J, Du C. Effect of electroacupuncture at Zusanli (ST36) on vagal electrical activity in the rat. Acupuncture Res. 2006;31 (5):290-293.

Yao T. Acupuncture and somatic nerve stimulation: mechanism underlying effects on cardiovascular and renal activities. Scand J Rehabil Med Suppl. 1993;29: 718, 1993. 